MINIREVIEW
Minireviews provides an opportunity to summarize existing knowledge of selected ecological areas, with special emphasis on current topics where rapid and significant advances are occurring. Reviews should be concise and not too wide-ranging. All key references should be cited. A summary is required.

\title{
Local mate competition, and extraordinary and ordinary blood parasite sex ratios
}

\author{
Dave Shutler and Andrew F. Read
}

\begin{abstract}
Shutler, D. and Read, A. F. 1998. Local mate competition, and extraordinary and ordinary blood parasite sex ratios. - Oikos 82: 417-424.

Blood parasites of the protozoan suborder Haemosporina include Plasmodium, Leucocytozoon, and Haemoproteus. Because life cycles of these parasites often lead to matings between individuals of the same genetic lineage (clones), selection for female-biased sex ratios should occur. Accordingly, sex ratios of populations of Plasmodium and Leucocytozoon closely matched expectations for probable rates of outbreeding. However, sex ratios of Haemoproteus populations were similar across a wide range of inferred outbreeding rates. Of several hypotheses we consider to explain this disparity, there are three we favour. The first is that the interaction among host immunity, parasite pathogenicity, and clone longevity differs among genera, so that on average more Haemoproteus clones coexist within hosts, thus reducing selection for female-biased sex ratios in that genus. The second hypothesis posits that hosts of the Haemoproteus populations we analysed had greater dispersal than did hosts of Plasmodium and Leucocytozoon. A consequence of this might be greater temporal and spatial variability in Haemoproteus outbreeding rates, so that Haemoproteus populations were prevented from achieving ideal sex ratios for the situation in which they found themselves. The third hypothesis is that Haemoproteus is vectored by relatively small midges so that biased parasite sex ratios in blood can result in too few gametes of one sex in a vector. Each hypothesis has its merits, and each could contribute to the patterns we observed. Our objective here is to stimulate further interest in the disparity.
\end{abstract}

D. Shutler, Dept of Biology, Univ. of Saskatchewan, 112 Science Place, Saskatoon, SK, Canada S7N 5E2 (shutlerd@desoto.wxe.sk.ec.gc.ca). - A. F. Read, Inst. of Cell, Animal and Population Biology, Univ. of Edinburgh, Edinburgh, UK EH9 $3 J T$.
Herein, we review our disparate results on sex allocation in blood parasites. Local mate competition (LMC; Hamilton 1967) should determine sex ratios in blood parasites. We begin by explaining why, and then summarise data from our almost identical studies which do (Read et al. 1995) and do not (Shutler et al. 1995) support this assertion. The bulk of this paper concerns hypotheses that may resolve the disparity. Our discussion centres on three genera of the protozoan suborder Haemosporina (Phylum Apicomplexa): Plasmodium, which includes species responsible for human malaria, and Leucocytozoon and Haemoproteus, which infect

Accepted 10 February 1998

Copyright (C) OIKOS 1998

ISSN 0030-1299

Printed in Ireland - all rights reserved 
birds. Most of our data concern the two avian genera, but much of the evidence comes from Plasmodium, because there is far more published information than for the two other genera. The Haemoproteus data provide the challenge to theory.

\section{Theoretical foundation and history}

Hamilton (1967) demonstrated that when a small number of mothers produce offspring that are to mate with each other, a female-biased offspring sex ratio is often favoured by natural selection. This situation is called LMC because a female-biased sex ratio reduces competition among brothers for mates (Taylor 1981). The optimum proportion of sons, $r^{*}$, is

$r^{*}=\frac{n-1}{2 n}$

where $n$ is the average number of mothers contributing to the mating pool. In the extreme case, where all mating is between sibs, females are predicted to produce just enough sons to mate all her daughters. LMC successfully explains many cases of extraordinary sex ratios among arthropod species, as well as facultative shifts in sex ratio within species (Hamilton 1967, Charnov 1982, Waage 1982, Wrensch and Ebbert 1993, Godfray 1994, Herre et al. 1997).

Plasmodium and many other haemosporidians also have life cycles that might lead to biased sex ratios through LMC (Ghiselin 1974, Pickering 1980, Schall 1989, Read et al. 1992). A quick review of these life cycles will clarify why. Within an infected vertebrate host, haploid macrogametocytes (equivalent to single eggs) and microgametocytes (equivalent to eight sperm) occupy erythrocytes. Seconds after a blood meal, ingested gametocytes release single macro- or up to eight microgametes within a fly vector's gut, and within a few minutes fertilisation occurs (Carter and Graves 1988, Atkinson and Van Riper 1991, Desser and Bennett 1993). Because gametes are drawn from a single vertebrate host, rather than the gamete population of many hosts, limited numbers of genetic lineages (clones) contribute to the mating pool within a vector, so that the frequency of within-clone mating (selfing) can be high. Various other bottlenecks in haemosporidian life histories (e.g., Fialho and Schall 1995) could also limit the number of clones reaching either vertebrate or fly hosts. In fact, population genetic analyses of Plasmodium falciparum populations have found averages of 1.3, 1.8, 2.3 and 3.3 clones per human host in Sudan, Papua New Guinea, The Gambia, and Tanzania, respectively (Hill and Babiker 1995, Hill et al. 1995, Paul et al. 1995, Babiker et al. 1997). In these circumstances, selfing will be common, so that female-biased sex ratios should be favoured. Read et al. (1992) give a more formal proof of this verbal argument (see also Dye and Godfray 1993, Read et al. 1995).

Two lines of evidence support the view that sex ratios in haemosporidian populations are a result of LMC. First, based on gametocyte sex ratios of $P$. falciparum in Papua New Guinea, Read et al. (1992) predicted that selfing rates in that population were between 60 and $100 \%$. Orthodoxy at that time, based on a failure to detect non-random associations between genes (linkage disequilibrium), was that Plasmodium populations were panmictic, with selfing rates close to zero (see Day et al. 1992 for review). Subsequent molecular genetic data revealed a selfing rate of $91 \%$ (Paul et al. 1995), consistent with the LMC prediction. Second, Read et al. (1995) reasoned that gametocyte prevalence (a measure of the proportion of hosts that are infectious) could be used to estimate the average number of infectious clones per person, and hence selfing rates, by assuming that the number of clones per host followed a negative binomial distribution. From this, they predicted a positive correlation between prevalence and gametocyte sex ratio. Data from 12 populations of Leucocytozoon and one of Plasmodium (Fig. 1) provided an excellent fit to this prediction.

Despite compelling support for LMC from Leucocytozoon, similar data for Haemoproteus (Shutler et al. 1995) did not accord with Read et al.'s (1995) prediction (Fig. 2). This is the disparity we seek to resolve.

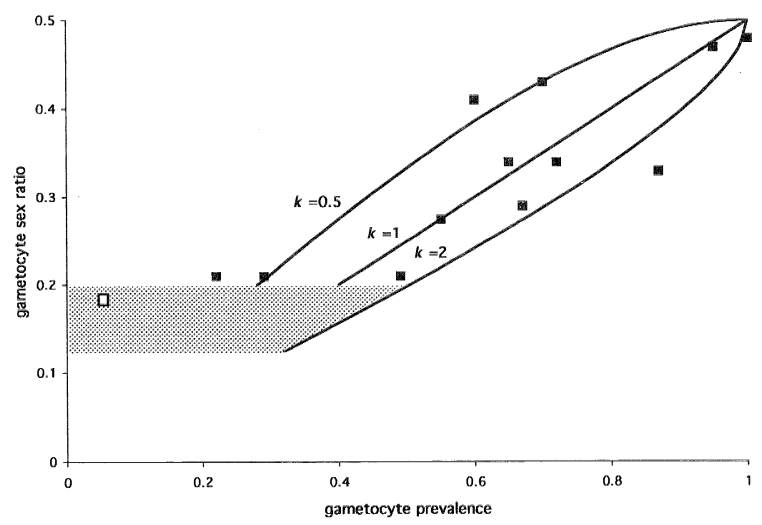

Fig. 1. Relationship between gametocyte sex ratio (proportion male) and gametocyte prevalence (proportion of hosts with gametocytes at detectable levels in peripheral blood). Plotted points are means for different host populations; filled squares Leucocytozoon spp.; open square, Plasmodium falciparum. Data from Read et al. (1995). Lines are the theoretical expectation with varying degrees of clonal aggregation, where $k$ is a parameter of the negative binomial distribution which is inversely related to the degree of aggregation (see Read et al. 1995 for model details and derivation). A lower limit (horizontal part of the curve) is expected where there are just sufficient male gametocytes to ensure fertilisation of all the female gametes. This depends on the average number of viable gametes released per male gametocyte, for which there is some uncertainty (shaded region). 


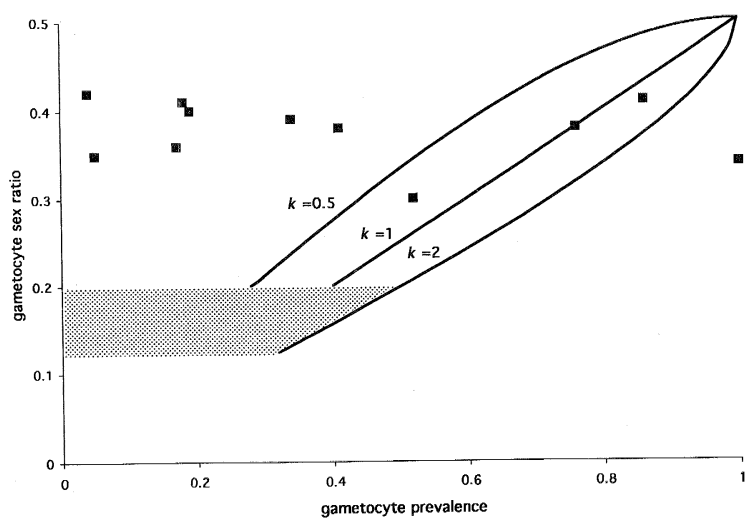

Fig. 2. Relationship between proportion of male Haemoproteus gametocytes and prevalence of infected hosts (data from Shutler et al. 1995). All points are means for different host populations. Further explanation in heading for Fig. 1.

\section{Hypotheses for the disparate results}

We first note that the disparity was not a consequence of different techniques used to determine sex ratios; some of both the Leucocytozoon and Haemoproteus data were assayed by A. Mullie and DS. Moreover, a few data from a different set of avian hosts (Anwar and Read unpubl.) had Haemoproteus sex ratios similar to those found by Shutler et al. (1995).

\section{The Shortage of Male Gametes Hypothesis}

Inefficiencies in microgametocyte formation can result in fewer than eight viable gametes per male gametocyte (Sinden 1983, Desser and Bennett 1993). If Haemoproteus microgametocyte division was less efficient than for the other genera, this could result in too few microgametes to fertilise available macrogametes, and result in more even gametocyte sex ratios. However, microgamete production per microgametocyte appears similar among genera (Bradbury and Trager 1968, Read et al. 1992).

\section{The Inertia Hypothesis}

This hypothesis is that genetic variation necessary for adaptive sex ratio evolution is absent (e.g., because sex is determined by Mendelian segregation of sex chromosomes; Williams 1979; also see Clutton-Brock 1986, Frank 1990, Koenig and Dickinson 1996). For it to explain our contrary results, the constraint on genetic variation would have to be exclusive to Haemoproteus. Although we cannot entirely discount this possibility, genetic details pertinent to sex ratio evolution are apparently identical for all Haemosporina (Carter and Graves 1988). Also, in Plasmodium at least, clones derived from a single parasite are capable of producing both micro- and macrogametocytes. Thus, sex determination can not be a consequence of Mendelian segregation of sex chromosomes. Actually, the only evidence that haemosporidian sex ratio is under at least some genetic control is the observation that sex ratios within Plasmodium clones stay constant over time (Burkot et al. 1984, Ranford-Cartwright et al. 1993, Taylor 1997, Read and Anwar unpubl.). Sex ratio could also be under environmental (proximate) control, because production of Plasmodium gametocytes is stimulated by environmental factors (Carter and Graves 1988, Buckling et al. 1997).

Regardless of the precise mechanisms of haemosporidian sex determination, there is significant inter-population variation in sex ratios within and among Leucocytozoon, Haemoproteus and Plasmodium (Schall 1989, Read et al. 1995, Shutler et al. 1995) on which selection could act. Moreover, selection among sex ratio variants within blood meals must be intense. For example, in crossing experiments between Plasmodium clones that differed in sex ratio of viable gametocytes, genetic representation in zygotes was greater for the least female-biased clone (Vaidya et al. 1993, 1995).

\section{The Lag Hypothesis}

Point estimates of avian blood parasite prevalence are notoriously variable through time and space (Cox 1989, Loye and Zuk 1991, Weatherhead and Bennett 1991, 1992, Weatherhead et al. 1991, Schall 1996, Shutler et al. 1996). Spot samples of prevalence may therefore provide relatively poor estimates of the selfing rates which imposed the selection responsible for the currently observed sex ratios. This is a highly plausible reason to expect Read et al.'s (1992 prediction to fail, and could account for the Haemoproteus data. But why then is the pattern sufficiently robust to be detectable in Leucocytozoon? We see two possibilities: (A) only Leucocytozoon is capable of facultative sex ratio adjustment in response to current conditions; (B) prevalence is more variable (spatially or temporally) for the sampled Haemoproteus populations, and this prevents maintenance of adaptive sex ratios. Both possibilities require selection among sampled Haemoproteus populations to vary in such a way as to promote a relatively narrow range of sex ratios $(0.30-0.42$ versus $0.18-0.48$ for Leucocytozoon and Plasmodium).

\section{3 (A) Facultative sex determination}

The Leucocytozoon data fit the LMC model despite temporal and spatial variability in prevalence, as is expected if parasites facultatively produce sex ratios adaptive for current probabilities of selfing. Facultative alteration requires that the parasite have the ability to predict the degree of selfing likely to be experienced (perhaps by recognising the relative density of their own gametocytes among all those in 
the blood), and be able to alter their sex ratio accordingly. (Note, however, that extreme temporal variation might prevent the parasite from keeping up; Charnov 1982.) Facultative control of sex ratio occurs among parasitoid wasps (Godfray 1994), but evidence is mixed for haemosporidians. Greater than binomial variation in sex ratios is found in Plasmodium populations ( Read et al. 1992, Robert et al. 1996, Pickering et al. unpubl.). This can occur if parasites are facultatively altering sex ratio in response to local conditions (Williams 1979). Moreover, sex ratios of some $P$. mexicanum infections can change dramatically over time (Schall 1989, 1996), which could reflect changing relatedness within infections. Correlations between gametocyte sex ratio and gametocyte density have been reported in two studies, and interpreted as facultative sex ratio adjustment, with parasites using gametocyte density as an indirect indicator of the number of clones within a host (Taylor 1997, Pickering et al. unpubl).

In contrast, two lines of evidence argue against facultative sex ratio control. Plasmodium clones maintained in laboratory monocultures in vivo and in vitro exhibit stable but often different sex ratios through time, and some even have 1:1 sex ratios (Trager et al. 1981, Burkot et al. 1984, RanfordCartwright et al. 1993, Vaidya et al. 1995, Taylor 1997, Read and Anwar unpubl.). If facultative sex ratio control occurred, all else being equal, singleclone infections should have the same female-biased sex ratio. Furthermore, recognition of the relative density of self gametocytes does not appear to occur; in experiments with laboratory mice, levels of relatedness within biclonal $P$. chabaudi infections did not influence sex ratios produced (Taylor 1997, Read and Anwar unpubl.). It is unclear whether absence of adaptive sex ratio shifts in laboratory clones is representative of wild-type parasites, or a consequence of chromosomal deletions that plague the Plasmodium genome in the laboratory (Kemp et al. 1990).

In any case, the conflicting pictures in Haemoproteus and Leucocytozoon (Figs. 1 and 2) could be reconciled if facultative sex ratio shifts, in response to the probability of selfing, occur in Leucocytozoon but not Haemoproteus, but there are currently no data to suggest such a difference.

\section{3 (B) Spatial and temporal variability in prevalence}

Intensity of LMC might be more poorly correlated with prevalence across some populations than across others. For instance, source populations (sensu Pulliam 1988) may have stable conditions that lead to relatively consistent, locally adaptive sex ratio optima, whereas sink populations may contain a mix of individuals that carry clones adapted to other areas. If more Haemoproteus than Leucocytozoon samples were obtained from hosts in sink populations, this may explain our disparity. Unfortunately, demographics of our populations are insufficiently characterised to test this hypothesis.

Extreme temporal variation may also prevent tight adaptation of sex ratio to prevalence. Prevalence of haemosporidian infections can vary substantially within and among years (Williams et al. 1980, Weatherhead and Bennett 1991, 1992, Schall 1996, Shutler et al. 1996). Genus-specific (see Williams et al. 1980) or sample population-specific differences in temporal variation could also explain our disparity. Atkinson and van Riper (1991) suggested that northern latitudes experience shorter and more intense bursts of haemosporidian transmission than do more southern latitudes. Longer seasons could create more predictable transmission rates and prevalence, leading to better fit to LMC theory of sex ratios. Resident birds are probably more likely to experience these conditions than are migrants. Six of 13 Leucocytozoon and Plasmodium species for which sex ratios were obtained came from resident host populations (Read et al. 1995), whereas only 1/11 Haemoproteus species was from a resident population (Shutler et al. 1995; Fisher's exact test, $P<0.05$ ). Mean absolute deviation from expectation (taking the clumping parameter $k=1$; see Read et al. 1995) of Haemoproteus sex ratios for 10 migratory populations was 0.22 , whereas it was only 0.02 for the single resident population ( $t$-test, $t=2.28, P=0.048)$. However, this pattern was not present in Leucocytozoon (mean absolute deviation was 0.05 in migrant populations compared with 0.03 in resident populations, $t=0.30$, $P=0.76$ ), and thus this explanation seems insufficient to resolve the disparity.

\section{The Avian Immunity Hypothesis}

Immune systems take time to become fully mature and active (Glick 1986, Baird 1995), and some haemosporidian infections elicit protective immunity (Fallis et al. 1951, Wernsdorfer and McGregor 1988). Consequently, older hosts may be better able to control or clear infections. This could mean that gametocyte prevalence in adults underestimates prevalence in younger hosts, where more transmission, and the bulk of selection on sex ratio, may occur. Thus, estimates of selfing rates based on gametocyte prevalences from adults could be too low, generating an apparently poor fit to LMC theory. This hypothesis could reconcile theory and data if adult clearance of Haemoproteus is pronounced, but is less so for Leucocytozoon. Clearance of these two genera between years did not differ among adults (Bennett and Bishop 1990), but the appropriate test would compare clearance rates between hatch-year and adulthood. Moreover, for three populations, sex ratios did not differ between hatch-year and adult birds (Shutler et al. 1995). 
In any case, why would one haemosporidian genus be cleared more rapidly from young birds than another? Leucocytozoon and Plasmodium are supposedly more pathogenic than Haemoproteus (Atkinson and Van Riper 1991, Desser and Bennett 1993). If Haemoproteus is less pathogenic because it is more successfully cleared by host immunity, this would accord with the Immunity Hypothesis. Alternatively, Haemoproteus could be less pathogenic because this genus distorts erythrocytes to a lesser extent than do the other two genera (Desser and Bennett 1993). Because immune responses involve fitness costs (resources, immunopathology; Behnke et al. 1992), selection should favour reduced responsiveness to benign parasites. If so, Haemoproteus should be less rapidly cleared, thus contradicting the Immunity Hypothesis. Clearly, direct measures of clearance rates from birds as they aged would be of considerable interest.

\section{The Unconventional Clumping Hypothesis}

Are Haemoproteus clones aggregated in hosts in a way that does not accord with the negative binomial model assumed by Read et al. (1995)? By invoking unexpected patterns of immunity, it may be possible to contrive statistical distributions that generate substantial aggregation in a small proportion of sampled hosts. For instance, if parasites are excluded from some hosts, but in others elicit relatively mild non-specific immunity, late-arriving clones could more easily invade and proliferate. The result would be that, even though prevalence was low, selfing would be uncommon, leading to less female-biased sex ratios. For this idea to reconcile data with theory, we would need evidence that Leucocytozoon and Haemoproteus provoke quantitatively different patterns of immune protection.

\section{The Unequal Investment Hypothesis}

Progeny sex ratios can deviate from 1:1 if one sex costs parents more to produce (Fisher 1950, Trivers and Hare 1976). This hypothesis, as it applies to haemosporidians, is dealt with and dismissed in detail by Read et al. (1992). Briefly, progeny parasites released from a vertebrate host cell (schizont), following clonal division of a parental parasite, either all become gametocytes or all remain as asexual parasites (Bruce et al. 1990). Parental investment therefore terminates when the schizont ruptures (schizogony). Relative cost of sons and daughters could be directly determined by relating the sex ratio resulting from a given schizont to the number of progeny it produces. That technically difficult experiment has not yet been done.

In lieu of assessing investment in progeny following schizogony, we can compare sexual size dimorphism of mature gametocytes. Haemoproteus macrogametocytes are generally smaller than microgametocytes (by up to 8\%; White and Bennett 1978, Bishop and Bennett 1989, 1990, Peirce et al. 1990). In contrast, Plasmodium and Leucocytozoon macrogametocytes are generally larger than microgametocytes (by up to 15\%; Fallis and Desser 1974, Schall 1989, Bennett et al. 1991, Bennett and Peirce 1992, Bennett and Squires-Parsons 1992). If dimorphism of mature gametocytes correlates with relative costs of producing them, these data predict that sex ratios of Haemoproteus should be more female-biased than those of the other two genera, which is opposite to what was observed (Figs. 1 and 2). However, even if dimorphism among earlier progeny stages was the reverse of that among mature gametocytes, the position of the predicted curves (Figs. 1 and 2) would be altered, but their shape would not. This too was not observed (Fig. 2).

\section{The Sex Ratio Distorter Hypothesis}

Many cases of biased sex ratios, in the absence of LMC or other conditions that should promote biases, have been traced to intracellular parasites that promote their own proliferation at the expense of their hosts (Werren et al. 1988). We cannot completely dismiss this hypothesis (Plasmodium has its own symbionts and extra-chromosomal elements; Wang and Wang 1991, Feagin 1994), but it is hard to imagine the sort of distorter that would result in a rather invariant sex ratio across widely divergent Haemoproteus populations, or that would produce sex ratios that, at equivalent prevalences, are less female-biased than those found in Leucocytozoon and Plasmodium. Moreover, addition of antibiotics to Plasmodium cultures has no effect on sex ratio (Read et al. 1992), suggesting that at least antibiotic-sensitive symbionts are not determining haemosporidian sex ratios.

\section{The Differential Mortality Hypothesis}

This hypothesis is that, before maturity, one sex of gametocyte suffers higher mortality than the other. This could result in apparently maladaptive sex ratios at the time of parasite breeding. Sex-specific immune clear ance is possible, although we are unaware of any published examples of sexual dimorphism in parasite antigens. At any rate, there is no evidence of sex-biased mortality of P. falciparum gametocytes (Smalley and Sinden 1977) and we have no a priori reason to expect that differential mortality would affect other haemosporidian genera dissimilarly.

\section{The Vector Size Hypothesis}

This hypothesis is that volume of blood meals influences the number of gametocytes ingested, and conse- 
quently the number of gametes available for fertilisation. (A variant, the Vector Environment Hypothesis, for which we have no data, posits that something inside the vector [e.g., gut morphology, immunity] makes it difficult for microgametes to find macrogametes.) Conditions that decrease the chance of a macrogamete being found by a microgamete concomitantly increase risks of producing surplus macrogametes, so that selection favours less female-biased sex ratios. In effect, this would increase the lower theoretical bound of the optimum proportion of males produced (Figs. 1 and 2). The existence of analogous constraints is well known for parasitoids and fig wasps (Green et al. 1982, Griffiths and Godfray 1988, Nagelkerke and Hardy 1994, Nagelkerke 1996, Herre et al. 1997, West et al. 1997). In support of the hypothesis, simple body size allometry suggests that midge (Ceratopogonidae) vectors of Haemoproteus take much smaller blood volumes than do black fly (Simuliidae) vectors of Leucocytozoon (Crosskey 1962, 1990) or mosquito (Anopheles) vectors of human Plasmodium (Clements 1992). Smaller midge blood volumes could constrain Haemoproteus sex ratios to stay closer to 1:1 (Fig. 2) to ensure fertilisation. Also consistent with this hypothesis is that species of lizard Plasmodium that are vectored by small phlebotomine flies typically have less female-biased sex ratios than does human $P$. falciparum, which is vectored by larger Anopheles mosquitoes (Schall 1989, 1996, Read et al. 1992, Robert et al. 1996). Hippoboscid flies can also transmit Haemoproteus (Valkiunas 1996), and it would be of considerable interest to determine sex ratios in populations where these relatively large flies are vectors.

Evidence against the Vector Size Hypothesis is that a greater proportion of erythrocytes may be occupied during Haemoproteus infections than during Leucocytozoon infections (Desser and Bennett 1993). This would increase the chances of Haemoproteus gametes finding each other in midge vectors, and would allow it to respond in the same way as Leucocytozoon to selection from LMC.

\section{Coda}

This review arose from our uncertainty about what caused our disparate results (Read et al. 1995, Shutler et al. 1995). We hope that the preceding stimulates other researchers to supply additional ideas and data. Currently we consider the Avian Immunity, Temporal and Spatial Variability, and Vector Size hypotheses to be leading contenders, though we disagree on their relative merits.

It is reasonable to ask why our disparity matters; sex ratio evolution is one of the most well-verified areas of evolutionary biology. First, precisely because of that, the Haemoproteus results are all the more interesting.
Second, the disparity may point to undiscovered biological features of these parasites, such as peculiarities in the dynamics of gamete or zygote formation. Third, if we can resolve the reasons for the disparity, we could apply LMC theory to disease treatment, because one could quickly assay genetic variation in haemosporidian populations using gametocyte sex ratios, obviating the need for costly, time-consuming, genetic techniques. Genetic relatedness within infections is thought to be a key parameter in determining the evolution of disease virulence (e.g., Anderson and May 1982, Nowak and May 1994). Finally, the application of evolutionary biology to infectious disease research, including much of what is optimistically called Darwinian Medicine (Williams and Nesse 1991, Ewald 1994, Nesse and Williams 1994), frequently involves optimality models (e.g. Levin and Pimental 1981, Anderson and May 1982). These normally assume equilibrium states, yet equilibrium is not an obvious feature of infectious diseases: rich epidemiological dynamics are expected and frequently observed (Anderson and May 1991, Nowak and May 1994). The success of sex allocation theory in the context of free-living organisms provides some of the best evidence in favour of the optimality approach to trait evolution. Similar tests on infectious disease organisms may give a good indication of the viability of optimality approaches to disease evolution.

Acknowledgements - We thank Alison Creasey, Adele Mullie, Lisa Ranford-Cartwright and Louise Taylor for bibliographic help, and Adele Mullie, Sean Nee, and Stuart West for comments and discussion. DS was knowingly but inadvertently supported by Robert G. Clark. AR is supported by a BBSRC Advanced Research Fellowship. We thank Oxford University and Mrs J. Braithwaite for making possible the summit that led to this culmination.

\section{References}

Anderson, R. M. and May, R. M. 1982. Coevolution of hosts and parasites. - Parasitology 85: 411-426.

Anderson, R. M. and May, R. M. 1991. Infectious diseases of humans: Dynamics and control. - Oxford Univ., Oxford.

Atkinson, C. T. and van Riper III, C. 1991. Pathogenicity and epizootiology of avian haematozoa: Plasmodium, Leucocytozoon, and Haemoproteus. - In: Loye, J. E. and Zuk, M. (eds), Bird-parasite interactions: ecology, evolution, and behaviour. Oxford Univ. Press, Oxford, pp. $19-48$.

Babiker, H. A., Lines, J., Hill, W. G. and Walliker, D. 1997. Population structure of Plasmodium falciparum in villages with different malaria endemicity in East Africa. - Am. J. Trop. Med. Hyg. 56: 141-147.

Baird, J. K. 1995. Host age as a determinant of naturally acquired immunity to Plasmodium falciparum. - Parasitol. Today 11: 105-111.

Behnke, J. M., Barnard, C. J. and Wakelin, D. 1992. Understanding chronic nematode infections: evolutionary considerations, current hypotheses and the way forward. - Int. J. Parasitol. 22: 861-907. 
Bennett, G. F. and Bishop, M. A. 1990. Change in status of haematozoan infections in wild passeriforms sampled in successive years. - Proc. Zool. Soc. (Calcutta) 43: 9-18.

Bennett, G. F. and Peirce, M. A. 1992. Leucocytozoids of 7 Old-World passeriform families. - J. Nat. Hist. 26: 693707.

Bennett, G. F. and Squires-Parsons, D. 1992. The leucocytozoids of the avian families Anatidae and Emberizidae, with descriptions of 3 new Leucocytozoon species. - Can. J. Zool. 70: 2007-2014.

Bennett, G. F., Earlé, R. A., Peirce, M. A., Huchzermeyer, F. W. and Squires-Parsons, D. 1991. Avian leucocytozoidae: The leucocytozoids of the phasianidae sensu lato. - J. Nat. Hist. 25: 1407-1428.

Bishop, M. A. and Bennett, G. F. 1989. The haemoproteids of the avian order Strigiformes. - Can. J. Zool. 67: 26762684.

Bishop, M. A. and Bennett, G. F. 1990. The hemoproteids of the avian families Corvidae (crows and jays) and Sturnidae (starlings and mynas) (Passeriformes). - Can. J. Zool. 68: 2251-2256.

Bradbury, P. C. and Trager, W. 1968. The fine structure of microgametogenesis in Haemoproteus columbae Kruse. - J. Protozool. 15: 700-712.

Bruce, M. C., Alano, P. and Carter, R. 1990. Commitment of the malaria parasite Plasmodium falciparum to sexual and asexual development. - Parasitology 100: 191-200.

Buckling, A. G. J., Taylor, L. H., Carlton, J. M.-R. and Read, A. F. 1997. Adaptive changes in Plasmodium transmission strategies following chloroquine chemotherapy. - Proc. R. Soc. Lond. B. 264: 553-559.

Burkot, T., Williams, J. L. and Schneider, I. 1984. Infectivity to mosquitos of Plasmodium falciparum clones grown in vitro from the same isolate. - Trans. R. Soc. Trop. Med. Hyg. 78: 339-341.

Carter, R. and Graves, P. M. 1988. Gametocytes. - In: Wernsdorfer, W. H. and McGregor, I. (eds), Malaria: principles and practice of malariology. Churchill Livingstone, Edinburgh, pp. 253-305.

Charnov, E. L. 1982. The theory of sex allocation. - Princeton Univ. Press, Princeton, NJ.

Clements, A. N. 1992. The biology of mosquitos, Vol. I. Development, nutrition, and reproduction. - Chapman and Hall, London.

Clutton-Brock, T. H. 1986. Sex ratio variation in birds. - Ibis 128: $317-329$.

Cox, F. E. G. 1989. Parasites and sexual selection. - Nature 341: 289.

Crosskey, R. W. 1962. Observations on the uptake of human blood by Simulium damnosum: the engorgement time and size of the blood meal. - Ann. Trop. Med. Parasitol. 56: $141-148$.

Crosskey, R. W. 1990. The natural history of blackflies. Wiley, London.

Day, K. P., Koella, J. C., Nee, S., Gupta, S. and Read, A. F. 1992. Population genetics and dynamics of Plasmodium falciparum: an ecological view. - Parasitology 104: S35S52.

Desser, S. S. and Bennett, G. F. 1993. The genera Leucocytozoon, Haemoproteus, and Hepatocystis. - In: Krier, J. P. (ed.), Parasitic protozoa, Vol. 4. Academic Press, New York, pp. 273-305.

Dye, C. and Godfray, H. C. J. 1993. On sex ratio and inbreeding in malaria parasite populations. - J. Theor. Biol. 161: 131-134.

Ewald, P. W. 1994. Evolution of infectious disease. - Oxford Univ. Press, Oxford.

Fallis, A. M. and Desser, S. S. 1974. On species of Leucocytozoon. - Adv. Parasitol. 12: 1-67.

Fallis, A. M., Davies, D. M. and Vickers, M. A. 1951. Life history of Leucocytozoon simondi Mathis and Leger in natural and experimental infections and blood changes produced in the avian host. - Can. J. Zool. 29: 305-328.
Feagin, J. E. 1994. The extrachromosomal elements of apicomplexan parasites. - Annu. Rev. Microbiol. 48: 81-104.

Fialho, R. F. and Schall, J. J. 1995. Thermal ecology of a malarial parasite and its insect vector: consequences for the parasite's transmission success. - J. Anim. Ecol. 64: 553562.

Fisher, R. A. 1950. The genetical theory of natural selection. Dover, London.

Frank, S. A. 1990. Sex allocation theory for birds and mammals. - Annu. Rev. Ecol. Syst. 21: 13-55.

Ghiselin, M. T. 1974. The economy of nature and the evolution of sex. - Univ. of California Press, Berkeley.

Glick, B. 1986. Immunophysiology. - In: Sturkie, P. D. (ed.), Avian physiology. Springer-Verlag, New York, pp. 87101.

Godfray, H. C. J. 1994. Parasitoids: behavioral and evolutionary ecology. - Princeton Univ. Press, Princeton, NJ.

Green, R. F., Gordh, G. and Hawkins, B. A. 1982. Precise sex ratios in highly inbred parasitic wasps. - Am. Nat. 120: 653-665.

Griffiths, N. and Godfray, H. C. J. 1988. Local mate competition, sex ratio and clutch size in bethylid wasps. - Behav. Ecol. Sociobiol. 22: 211-217.

Hamilton, W. D. 1967. Extraordinary sex ratios. - Science 156: $477-488$.

Herre, E. A., West, S. A., Cook, J. M., Compton, S. G. and Kjellberg, F. 1997. Fig wasp mating systems: pollinators and parasites, sex ratio adjustment and male polymorphism, population structure and its consequences. - In: Choe, J. and Crespi, B. (eds), Social competition and cooperation in insects and arachnids. In: The evolution of mating systems, Vol. I. Princeton Univ. Press, Princeton, NJ, pp. 226-239.

Hill, W. G. and Babiker, H. A. 1995. Estimation of numbers of malaria clones in blood samples. - Proc. R. Soc. Lond. B. 262: 249-257.

Hill, W. G., Babiker, H. A., Ranford-Cartwright, L. C. and Walliker, D. 1995. Estimation of inbreeding coefficients from genotypic data on multiple alleles, and application to estimation of clonality in malaria parasites. - Genet. Res. 65: $53-61$.

Kemp, D. J., Cowman, A. F. and Walliker, D. 1990. Genetic diversity in Plasmodium falciparum. - Adv. Parasitol. 29: $75-149$.

Koenig, W. D. and Dickinson, J. L. 1996. Nestling sex ratio variation in Western Bluebirds. - Auk 113: 902-910.

Levin, S. and Pimental, D. 1981. Selection of intermediate rates of increase in host-parasite systems. - Am. Nat. 117: $308-315$

Loye, J. E. and Zuk, M. 1991. Bird-parasite interactions. Oxford Univ. Press, Oxford.

Nagelkerke, C. J. 1996. Discrete clutch sizes, local mate competition, and the evolution of precise sex allocation. Theor. Popul. Biol. 49: 314-343.

Nagelkerke, C. J. and Hardy, I. C. W. 1994. The influence of developmental mortality on optimal sex allocation under local mate competition. - Behav. Ecol. 5: 401-411.

Nesse, R. M. and Williams, G. C. 1994. Why we get sick. Time Books, New York.

Nowak, M. A. and May, R. M. 1994. Superinfection and the evolution of parasite virulence. - Proc. R. Soc. Lond. B. 255: $81-89$.

Paul, R. E. L., Packer, M. J., Walmsley, M., Lagog, M., Ranford-Cartwright, L. C., Paru, R. and Day, K. P. 1995. Mating patterns in malaria parasite populations of Papua New Guinea. - Science 269: 1709-1711.

Peirce, M. A., Bennett, G. F. and Bishop, M. A. 1990. The hemoproteids of the avian order Falconiformes. - J. Nat. Hist. 24: 1091-1100.

Pickering, J. 1980. Sex ratio, social behaviour and ecology in Polistes (Hymenoptera, Vespidae), Pachysomoides (Hymenoptera, Ichneumonidae) and Plasmodium (Protozoa, Haemosporidae). - Ph.D. thesis, Harvard Univ. 
Pulliam, H. R. 1988. Sources, sinks, and population regulation. - Am. Nat. 132: 652-661.

Ranford-Cartwright, L. C., Balfe, P., Carter, R. and Walliker, D. 1993. Frequency of cross-fertilisation in the human malaria parasite Plasmodium falciparum. - Parasitology 107: $11-18$.

Read, A. F., Narara, A., Nee, S., Keymer, A. E. and Day, K. P. 1992. Gametocyte sex ratios as indirect measures of outcrossing rate in malaria. - Parasitology 104: 387-395.

Read, A. F., Anwar, M., Shutler, D. and Nee, S. 1995. Sex allocation and population structure in malaria and related parasitic protozoa. - Proc. R. Soc. Lond. B. 260: 359-363.

Robert, V., Read, A. F., Essong, J., Tchuinkam, T., Mulder, B., Verhave, J.-P. and Carnevale, P. 1996. Effect of gametocyte sex ratio on infectivity of Plasmodium falciparum to Anopheles gambiae. - Trans. R. Soc. Trop. Med. Hyg. 90: 621-624.

Schall, J. J. 1989. The sex ratio of Plasmodium gametocytes. Parasitology 98: 343-350.

Schall, J. J. 1996. Malarial parasites of lizards: diversity and ecology. - Adv. Parasitol. 37: 255-333.

Shutler, D., Bennett, G. F. and Mullie, A. 1995. Sex proportions of Haemoproteus blood parasites and local mate competition. - Proc. Natl. Acad. Sci. USA 92: 6748-6752.

Shutler, D., Ankney, C. D. and Dennis, D. G. 1996. Could the blood parasite Leucocytozoon deter mallard range expansion? - J. Wildl. Manage. 60: 569-580.

Sinden, R. E. 1983. Sexual development of malarial parasites. - Adv. Parasitol. 22: 153-216.

Smalley, M. E. and Sinden, R. E. 1977. Plasmodium falciparum gametocytes: their longevity and infectivity. - Parasitology 74: $1-8$

Taylor, P. D. 1981. Intra-sex and inter-sex sibling interactions as sex ratio determinants. - Nature 291: 64-66.

Taylor, L. H. 1997. Epidemiological and evolutionary consequences of mixed-genotype infections of malaria parasites. - D.Phil., Univ. of Edinburgh.

Trager, W., Tershakovec, M., Lyandvert, L., Stanley, H., Lanners, N. and Gubert, E. 1981. Clones of the malaria parasite Plasmodium falciparum obtained by microscopic selection: their characterisation with regard to knobs, chloroquine sensitivity, and formation of gametocytes. Proc. Natl. Acad. Sci. USA 78: 6527-6530.

Trivers, R. L. and Hare, H. 1976. Haplodiploidy and the evolution of the social insects. - Science 191: 249-263.

Vaidya, A. B., Morrisey, J., Plowe, C. V., Kaslow, D. C. and Wellems, T. E. 1993. Unidirectional dominance of cyto- plasmic inheritance in two genetic crosses of Plasmodium falciparum. - Mol. Cell. Biol. 13: 7349-7357.

Vaidya, A. B., Muratova, O., Guinet, F., Keister, D., Wellems, T. E. and Kaslow, D. C. 1995. A genetic locus on Plasmodium falciparum chromosome 12 linked to a defect in mosquito infectivity and male gametocytogenesis. Mol. Biochem. Parasitol. 69: 65-71.

Valkiunas, G. 1996. Ecological implications of hematozoa in birds. - Bull. Scand. Soc. Parasitol 6: 103-113.

Waage, J. K. 1982. Sib-mating and sex ratio strategies of scelionid wasps. - Ecol. Entomol. 7: 103-112.

Wang, A. L. and Wang, C. C. 1991. Viruses of parasitic protozoa. - Parasitol. Today 7: 76-80.

Weatherhead, P. J. and Bennett, G. F. 1991. Ecology of red-winged blackbird parasitism by haematozoa. - Can. J. Zool. 69: 2352-2359.

Weatherhead, P. J. and Bennett, G. F. 1992. Ecology of parasitism of brown-headed cowbirds by haematozoa. Can. J. Zool. 70: 1-7.

Weatherhead, P. J., Bennett, G. F. and Shutler, D. 1991. Sexual selection and parasites in wood-warblers. - Auk 108: $147-152$.

Wernsdorfer, W. H. and McGregor, I. (eds) 1988. Malaria: Principles and practice of malariology. - Churchill Livingstone, Edinburgh.

Werren, J. H., Nur, U. and Wu, C.-I. 1988. Selfish genetic elements. - Trends Ecol. Evol. 3: 297-302.

West, S. A., Herre, E. A., Compton, S. G., Godfray, H. C. J. and Cook, J. M. 1997. A comparative study of virginity in fig wasps. - Anim. Behav. 54: 437-450.

White, E. M. and Bennett, G. F. 1978. Avian Haemoproteidae. 9. Description of Haemoproteus stellaris $\mathrm{n}$. sp. and a review of the haemoproteids of the swallow family Hirundinidae. - Can. J. Zool. 56: 2110-2116.

Williams, G. C. 1979. The question of adaptive variation in sex ratio in out-crossed vertebrates. - Proc. R. Soc. Lond. B. 205: $567-580$.

Williams, G. C. and Nesse, R. M. 1991. The dawn of Darwinian medicine. - Q. Rev. Biol. 66: 1-22.

Williams, N. A., Mahrt, J. L. and Zwickel, F. C. 1980. The ecology of blood parasites in blue grouse from Vancouver Island, British Columbia. - Can. J. Zool. 58: 21752186.

Wrensch, D. L. and Ebbert, M. A. 1993. Evolution and diversity of sex ratio in insects and mites. - Chapman and Hall, New York. 